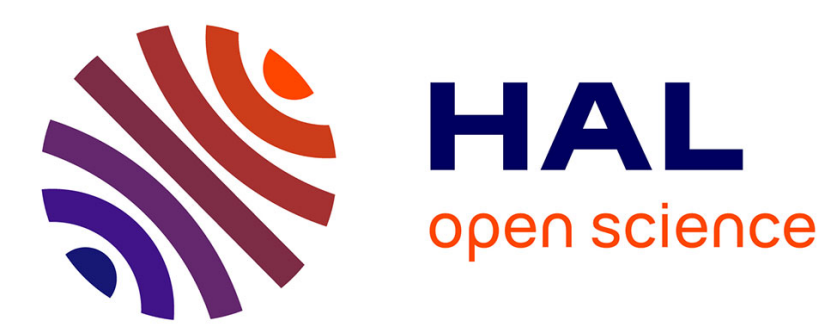

\title{
Elastic behavior of composites containing Boolean random sets of inhomogeneities
}

François Willot, Dominique Jeulin

\section{To cite this version:}

François Willot, Dominique Jeulin. Elastic behavior of composites containing Boolean random sets of inhomogeneities. International Journal of Engineering Science, 2009, 47 (2), pp.313-324. 10.1016/j.ijengsci.2008.09.016 . hal-00426398

\section{HAL Id: hal-00426398}

https://hal-mines-paristech.archives-ouvertes.fr/hal-00426398

Submitted on 20 Feb 2014

HAL is a multi-disciplinary open access archive for the deposit and dissemination of scientific research documents, whether they are published or not. The documents may come from teaching and research institutions in France or abroad, or from public or private research centers.
L'archive ouverte pluridisciplinaire HAL, est destinée au dépôt et à la diffusion de documents scientifiques de niveau recherche, publiés ou non, émanant des établissements d'enseignement et de recherche français ou étrangers, des laboratoires publics ou privés. 


\title{
Elastic behavior of composites containing Boolean random sets of inhomogeneities
}

\author{
François Willot*, Dominique Jeulin \\ MINES Paristech, Centre de Morphologie Mathématique, \\ Mathématiques et Systèmes, \\ 35, rue Saint Honoré, 77305 Fontainebleau CEDEX, France.
}

\begin{abstract}
The overall mechanical response as well as strain and stress field statistics of an heterogeneous material made of two randomly distributed, linear-elastic phases, are investigated numerically. The Boolean model of spheres is used to generate microstructures consisting of either porous or rigid inclusions, at any volume fraction of the phases. Stress and strain field integral ranges, or equivalently the representative volume element, are computed and linked to features of the field statistics, and to the microstructure geometry.
\end{abstract}

Key words: Homogenization, Bulk modulus, Representative volume element, Integral range, Boolean model of spheres, Linear elasticity, Random media PACS: 46.04.+b,46.15.-x,46.65.+g

\section{Introduction}

This work is concerned with the overall (i.e. mean) elastic response and field statistics of an heterogeneous medium subjected to an applied macroscopic deformation. The usual approach for the prediction of the elastic properties of random media is based on the calculation of bounds from a limited amount of information on the microstructure. Hashin and Shtrikman (9) rely on a specific variationnal principle and on the assumption of statistical morphological isotropy of the medium. Beran and Molyneux (1) worked out third order bounds of the bulk modulus based on the three-points statistics of random

\footnotetext{
* Corresponding author.

Email addresses: francois.willot@cmm.ensmp.fr (François Willot), dominique.jeulin@cmm.ensmp.fr (Dominique Jeulin).
} 
media. These bounds were specialised to the case of two-phase media by Milton $(21)$, and to some specific models of random media $(26 ; 10)$. However, bounds are of limited usefulness when the contrast between components of an heterogeneous medium is too large (namely for porous media or for rigid components). In the absence of any general analytical formula to predict the overal elastic properties of random media, an alternative way is based on the use of numerical techniques to solve the elasticity problem on images of the microstructure. Recent progress in computer science and in numerical implementation of the solution of partial differential equations make it possible to operate on 3D images. However, the size of the images that can be handled still remains (and will remain) limited, so that must be addressed the question of the representativity of the effective properties estimated by this approach $(14 ; 11 ; 13)$.

In this paper, we consider the numerical homogenization of the bulk modulus of the Boolean model of spheres, in the case of porous or rigid inclusions. We first give a short reminder of the Boolean model and of its periodic version. In a second step, the numerical technique used to estimate the stress field, based on iterations of Fast Fourier transforms, is detailed. It is validated in the case of a periodic array of spheres, for which an accurate estimate is known $(3 ; 4)$. Results obtained for Boolean models of spheres, covering the effective properties as well as some statistical properties of the field are given in the case of porous and rigid inclusions.

\section{The Boolean model of spheres in linear elasticity}

\subsection{General framework}

This study is focused on a medium made of two linear-elastic phases, which consists of spherical overlapping inclusions (phase 2) embedded in a "matrix" (phase 1). The limit of infinitely-contrasted properties between the phases is considered, i.e. the material is either reinforced with rigid particles or weakened by pores. More precisely, the microstructure is generated from a Boolean model of spheres (18), with the number of sphere centers in any given region following a Poisson point process. The intensity of the Poisson process is chosen so that the volume fraction of the inclusions is equal, on average, to the prescribed volume fraction. Consequently, the volume fraction is different for each configuration, except in the limit of an infinite sample.

This work is restricted to the case of an isotropic macroscopic deformation, which yields results pertaining to the effective bulk modulus, whereas the effective shear modulus is, on average, null. It is assumed that the applied loading 
is small enough that it induces very small matter displacement (compared to the smallest typical length of the microstructure), such that the phase geometry may be fixed for each given sample, and the homogenization problem be treated within the theory of small perturbations.

Given a finite sample of the medium, materialized by a cube of size $L$, an overall strain loading with periodic boundary conditions (defined below) is applied. Previous results (15) obtained for random linear composites have shown that, as far as the effective elastic moduli are concerned, faster size convergence is achieved with periodic boundary conditions than with classical "kinematic" or "static" types boundary conditions, which tend to perturbate fields along boundaries. To take advantage of this property, a Boolean model suited to periodic media is used i.e. each randomly-chosen sphere center, or seed, is periodicized before being replaced with spheres.

\subsection{Constitutive law}

The constitutive law relates at each point $\mathbf{x}$ in the medium the stress and strain tensors $\sigma$ and $\varepsilon$. In the matrix, it is characterized by its bulk and shear elastic moduli, noted repectively $\kappa$ and $\mu$, and is defined by:

$$
\sigma(\mathbf{x})=\mathbb{L}: \varepsilon(\mathbf{x}), \quad L_{i j k l}=\kappa \delta_{i j} \delta_{k l}+\mu\left(\delta_{i k} \delta_{j l}+\delta_{i l} \delta_{j k}\right)
$$

where $\mathbb{L}$ is a fourth-order elastic tensor, the double point : denotes a double contraction between its right and left-hand terms, and $\delta$ is the Kronecker symbol. In the following, the shear and bulk moduli in the matrix have been chosen as: $\kappa=1 / 3, \mu=1 / 2$. It is assumed that the results of this work apply qualitatively to more general materials as long as both elastic moduli in the matrix are finite. Expanded in terms of the mean and deviatoric strain and stress components:

$$
\sigma_{m}=3 \kappa \varepsilon_{m}, \quad \sigma^{\prime}=2 \mu \varepsilon^{\prime}, \quad \varepsilon_{i j}^{\prime}=\varepsilon_{i j}-\varepsilon_{m} \delta_{i j}, \quad \sigma_{i j}^{\prime}=\sigma_{i j}-\sigma_{m} \delta_{i j},
$$

where the mean strain and stress are the scalars noted $\varepsilon_{m}=\varepsilon_{i i} / 3$ and $\sigma_{m}=$ $\sigma_{i i} / 3$, respectively, whereas the deviatoric parts $\varepsilon^{\prime}$ and $\sigma^{\prime}$ are tensors. In phase 2 , the constitutive law in porous (resp. rigid) inclusions is $\sigma \equiv 0$ (resp. $\varepsilon \equiv 0$ ) with strain (resp. stress) undefined. 


\subsection{Stress equilibrium and strain compatibility}

The strain must derive from a displacement field $\mathbf{u}$ (referred to as the "strain compatibility" condition). Assuming small deformation, this reads:

$$
\varepsilon_{i j}=\frac{1}{2}\left(\delta_{i} u_{j}+\delta_{j} u_{i}\right)
$$

Symetrically, the stress field satisfies an "equilibrium equation", which reads, in the absence of external forces:

$$
\partial_{i} \sigma_{i j}=0
$$

Note that interfaces between inclusions and matrix are perfect, i.e. the stress boundary conditions along matrix-pores interfaces read

$$
\sigma \cdot \mathbf{n}=0
$$

where $\mathbf{n}$ is a vector normal to the boundary. In the case of a rigid inclusion, the boundary condition along interfaces read, in terms of the displacement field:

$$
\mathbf{u} \cdot \mathbf{n}=0,
$$

Assuming sufficiently smooth interfaces (i.e. without angles or corners), Eq. (5) may be derived using (4), (3), writing each derivative as a small difference between two points accross the interface. However, in the numerical computations undertaken in this work, it is easier to appeal to the constitutive law and to the equilibrium and compatibility equations, defined at each point in the heterogeneous material, rather than interface boundary conditions.

\section{Full-fields computations by means of Fast Fourier Transforms}

The "Fast Fourier method" introduced by Moulinec, Suquet (22) is a breakthrough in numerical methods for computing the mechanical response of composite materials. It has successfully been applied to many other homogenization problems, e.g. conductivity or permittivity (13)). It makes use of an iterative fixed-point algorithm derived from the periodic Lippman-Shwinger equation (see e.g. Kroner (16)). Given an homogeneous elastic reference material of elastic tensor $\mathbb{L}^{0}$, the strain field $\varepsilon$ is a solution of the implicit equation:

$$
\varepsilon(\mathbf{x})=\langle\varepsilon\rangle-\int \mathrm{d}^{3} \mathbf{x}^{\prime} \mathbb{G}^{0}\left(\mathbf{x}-\mathbf{x}^{\prime}\right): \tau\left(\mathbf{x}^{\prime}\right), \quad \tau(\mathbf{x})=\sigma(\mathbf{x})-\mathbb{L}^{0}: \varepsilon(\mathbf{x}),
$$

where the Green function $\mathbb{G}^{0}$ depends only on the reference tensor $\mathbb{L}^{0}$, and its average is chosen equal to zero. The polarization field with respect to the 
reference $\mathbb{L}^{0}$ is noted $\tau$ and $\langle\varepsilon\rangle$ is the prescribed macroscopic strain field. The original scheme of the FFT method is a straightforward application of the Lippman-Schwinger equation. Given an image of the strain field (e.g. $\varepsilon \equiv$ 0 ), the polarization field $\tau$ on the right side equation in (7) is computed at each point in the real space, making use of the constitutive law, whereas the convolution on the left side equation is computed in the Fourier space, as a product, yielding a new strain field. The original FFT algorithm consists in iterating the two equations, computing forward and backward fast Fourier transforms at each step, on a cubic grid of $L \times L \times L$ voxels. Iterations are stopped when the given convergence criterion is lower than a fixed value. Authors commonly check for stress equilibrium in the Fourier space e.g.:

$$
\frac{\left[\sum_{\mathbf{q}}|\mathbf{q} \cdot \hat{\sigma}(\mathbf{q})|^{2}\right]^{1 / 2}}{|\hat{\sigma}(0)|}<\eta,
$$

where $\eta$ is typically of order $10^{-5}$. The use of an optimal reference elastic tensor $\mathbb{L}^{0}$ strongly improves convergence, however the number of iterations to achieve convergence is roughly proportional to the contrast between the phases, so that this method can not be applied to infinitely contrasted (e.g. porous) media.

Subsequently, Eyre and Milton (8) and Michel et al. (20) have developped refined algorithms which proved more efficient in the case of a high contrast between the phases (see Moulinec, Suquet (24) for comparisons between the three methods). In this work, we use Michel's "agmented Lagrangian" algorithm, which, among its advantage, is the only one to converge in the limit of an infinitely contrasted medium. However, no formula for the optimum elastic reference tensor $\mathbb{L}^{0}$ is known. Worst, numerical computations show that the optimum choice is very sensitive to the contrast, geometry and the required precision, although less to the system size. Consequently, a sub-optimal reference field is numerically estimated at small grid sizes, and applied to larger systems.

The Augmented Lagrangian scheme is derived from the minimization of an elastic potential over an auxiliary variable e with the additional condition that $\mathbf{e}=\varepsilon$ (see Eq. (6) in Moulinec, Suquet (24)). Contrary to the strain field $\varepsilon$ in the the original scheme, the compatibuility for $\mathbf{e}$ is not enforced at each step. Since the Fourier transform of the stress is not directly available in the augmented Lagrangian algorithm, convergence is checked as a relative difference in the real space between the tensors $\varepsilon$ and $\mathbf{e}$ :

$$
\left(\sum_{\mathbf{x}}\|\varepsilon(\mathbf{x})-\mathbf{e}(\mathbf{x})\|^{2}\right)^{1 / 2} /\|\hat{\sigma}(0)\|<\eta
$$

with $\eta=10^{-6}$ or $\eta=10^{-7}$. Attention must be paid to the highest frequencies 
(e.g. $q= \pm(L / 2+1)$, where $L$ is the number of voxels along each direction, see Moulinec, Suquet (23)), at which point the Green function lacks its usual symmetry (i.e. $\mathbb{G}^{*}(\mathbf{q}) \neq \mathbb{G}(-\mathbf{q})$ ), inducing an edge effect. To check this effect, we have compared the strain fields as computed with two different methods "FFT-I" and "FFT-II" that ensure, respectively, $\hat{\sigma}(\mathbf{q})=0$ and $\mathbb{G}(\mathbf{q})=0$ at the highest modes. In Fig. 1, both numerical methods are compared to 2D exact solutions recently derived (Willot, Pellegrini, Ponte (27)) in the case of a strongly anisotropic medium weakened by an array of porous cylinders. As expected, the difference between results obtained with the two methods "FFT-I" and "FFT-II" is negligible compared to the size effect. The exact solution is recovered when size increases, with high oscillations observed at low resolution.

In the case of a rigid phase, high but finite values of the local elastic moduli must be introduced. In the numerical computations, we have chosen $\kappa^{\prime}=\mu^{\prime}=$ $10^{3} \gg \kappa, \mu$ as the local bulk and shear moduli in "rigid" inclusions. In pores, however, $\kappa^{\prime}=\mu^{\prime}=0$.

\section{Homogenization and overall loading}

Given a sufficiently large sample of a microstructure, the material behavior is governed by effective properties which relate the macroscopic strain and stress tensors. When all phases are locally linear elastic, the effective constitutive law is also linear elastic:

$$
\langle\sigma\rangle=\mathbb{L}_{0}:\langle\varepsilon\rangle
$$

where $\langle\cdot\rangle$ denotes an average over the sample. Conversely, a given sample of a material is said to be statistically representative of a microstructure if it is large enough to satisfy Eq. (10). Consistently with Eq. (10), we apply a macroscopic strain field $\varepsilon_{0}=\langle\varepsilon\rangle$ in a given direction, and derive the effective elastic tensor from the resulting macroscopic stress field $\sigma_{0}=\langle\sigma\rangle$. Averages are computed from the complete images of the strain and stress fields that satisfy periodic boundary conditions, equilibrium and compatibility as well as the microscopic strain-stress law.

\section{$5 \quad$ Validation on a periodic array of spheres}

In this section, we investigate the accuracy of the FFT method against an analytical approximation (3) of the effective elastic moduli of a cubic array of spheres which draws on previously developped methods by $\operatorname{Bergman}(2)$. This estimate gives, in terms of the sphere volume fraction $f$, an analytical 
formula for the effective bulk modulus which is valid up to order 3 in $f$. First, the effective elastic moduli is numerically computed at a high level of accuracy, up to 8 digits, for finite contrast, with the FFT tool (we have chosen elastic moduli in the spheres that are two times higher than in the matrix). Comparing these results with analytical estimates, we have checked that the difference between the Bergman and FFT computations is of order $\sim f^{4}$ (see Fig. 2). Secondly, at infinite contrast (here, with porous inclusions), the Bergman estimate compares well to FFT results for the effective bulk and elastic shear modulus (Fig. 3).

\section{Results}

\subsection{Effective moduli}

In this section and hereafter, the Boolean model of spheres is considered for the microstructure. The effective bulk modulus as a function of the fraction $f$ of porous and rigid inclusions are given in Figs. (4) and (5). For comparison the self consistent [SC] estimates, as well as standard Hashin and Shtrikman [HS] bounds, and tighter third-order Beran bounds [TOB] are provided. It appears that in the present case, bounds are not useful, being too much separated. In addition, the self-consistent estimate, does not provide a pertinent estimate over the entire range of volume fractions.

The effects of percolation appear clearly on these figures: the bulk modulus vanishes for a pore volume fraction at $f=f^{\prime} \approx 0.95$ in Fig. (4). According to various studies the geometrical percolation threshold of the matrix of a Boolean model ranges from 0.946 (12) to 0.97 (7). In the porous Boolean medium, we observe the scaling law: $\kappa_{0} / \kappa \sim 6.5\left(f^{\prime}-f\right)^{3.0}$ (see Fig. 4), for $\left|f^{\prime}-f\right|<0.1$.

Inversely, in the rigid Boolean model, the bulk modulus grows according to a power law beyond the percolation threshold (close to $f=f^{\prime \prime} \approx 0.29$, see e.g. $(25 ; 12)$ ). It turns out that our results for $f>f^{\prime \prime}$ may be fited with a good approximation to a power law dependence on $f$, for almost the full range of volume fractions beyond percolation. Normalizing the effective bulk modulus with the bulk modulus in the spheres, we find $\kappa_{0} / \kappa_{2} \approx 2.39\left(f-f^{\prime \prime}\right)^{2.35}$ for $f>f^{\prime \prime} \approx 0.29$. Interestingly, power law dependencies that extend well beyond the percolation region have been found(6) for the Young modulus of a different medium. 


\subsection{Fluctuations of effective properties}

In this section the representativity of the effective property estimated on a bounded domain of a microstructure is addressed. To compute the so-called "Representative Volume Element" $(14 ; 11)$ (RVE) we make use of a geostatistical approach based on the experimental determination of the integral range (17) from numerical simulations.

\subsubsection{The integral range}

We consider fluctuations of average values over different realizations of a random medium inside the domain $B$ with the volume $V$. In geostatistics, it is well known that for an ergodic stationary random function $Z(x)$, with mathematical expectation $E(Z)$, one can compute the variance $D_{Z}^{2}(V)$ of its average value $\bar{Z}(V)$ over the volume $V$ as a function of the central covariance function $\bar{C}(h)$ of $Z(x)(17)$ by :

$$
D_{Z}^{2}(V)=\frac{1}{V^{2}} \int_{V} \int_{V} \bar{C}(x-y) d x d y
$$

where

$$
\bar{C}(h)=E\{(Z(x)-E(Z))(Z(x+h)-E(Z))\}
$$

For a large specimen (with $V \gg A_{3}$ ), equation (11) can be expressed to the first order in $1 / V$ as a function of the integral range in the space $R^{3}, A_{3}$, by

$$
\begin{aligned}
D_{Z}^{2}(V) & =D_{Z}^{2} \frac{A_{3}}{V} \\
\text { with } A_{3} & =\frac{1}{D_{Z}^{2}} \int_{R^{3}} \bar{C}(h) d h
\end{aligned}
$$

where $D_{Z}^{2}$ is the point variance of $Z(x)$ (computed by taking averages from numerical estimates) and $A_{3}$ is the integral range of the random function $Z(x)$, defined when the integral in equations (11) and (13) is finite. The asymptotic scaling law (12) is valid for an additive variable $Z$ over the region of interest $V$.

In the case of a two-phase material modeled by a random set $A$ with the characteristic function $k(x)$ (with the volume fraction $p$ ), the point variance $D_{Z}^{2}$ of the random function $k(x)$ is given by $p(1-p)$, and the central covariance function is obtained from the set covariances $C(h)=P\{x \in A, x+h \in A\}$ or $Q(h)=P\left\{x \in A^{c}, x+h \in A^{c}\right\}$ by :

$$
\bar{C}(h)=C(h)-p^{2}=Q(h)-q^{2},
$$

with $q=1-p$. 
To estimate the effective elasticity tensor $\mathbb{L}_{0}$ defined by Eq. 10, we have to compute the averages $\langle\sigma\rangle$ or $\langle\varepsilon\rangle$. For the applied boundary conditions (a hydrostatic loading in the present case), the bulk modulus is obtained from the estimations of a scalar, namely the mean stress field. Therefore the variance of the effective property $\kappa_{0}$ follows the equation (12) when the integral range $A_{3}$ of the relevant field is known.

Since the theoretical covariance of the fields ( $\sigma$ or $\varepsilon$ ) is not available, the integral range can be estimated according to the procedure proposed by Georges Matheron for any random function (19): working with realizations of $Z(x)$ on domains $B$ with an increasing volume $V$ (or in the present case considering subdomains of large simulations, with a wide range of sizes), we can estimate the parameter $A_{3}$ by fitting the obtained variance according to the expression (12). The point variance $D_{Z}^{2}$ of the corresponding field is directly estimated from the experimental variance of the field.

\subsubsection{Practical determination of the size of the RVE}

When considering a material as a realization of a random set or of a random function RF, the idea that there exists one single possible minimal RVE size must be left out. Instead, the size of a RVE can be defined for a physical property $Z$, a contrast and, above all, a given precision in the estimation of the effective properties depending on the number of realizations that one is ready to generate. By means of a standard statistical approach, the absolute error $\epsilon_{a b s}$ and the relative error $\epsilon_{\text {rela }}$ on the mean value obtained with $n$ independent realizations of volume $V$ are deduced from the interval of confidence by:

$$
\epsilon_{a b s}=\frac{2 D_{Z}(V)}{\sqrt{n}} ; \epsilon_{\text {rela }}=\frac{\epsilon_{a b s}}{Z}=\frac{2 D_{Z}(V)}{Z \sqrt{n}}
$$

The size of the RVE can now be defined as the volume for which for instance $n=1$ realization is necessary to estimate the mean property $Z$ with a relative error $\epsilon_{\text {rela }}=1 \%$, provided that the function $D_{Z}(V)$ is known. Alternatively, we can decide to operate on smaller volumes (provided no bias is introduced), and consider $n$ realizations to obtain the same relative error. In the case of effective elastic moduli, the exact mean value and variance for a given domain size are a priori unknown. The absolute error on the mean value is evaluated using Eq. (14). This methodology was applied to the case of the dielectric permittivity of various random media $(5 ; 13)$, and to the elastic properties and thermal conductivity of a Voronoï mosaic (14). 


\subsubsection{Covariances and integral range for the Boolean model}

In this section, we consider the covariance field of the characteristic function of phase (2) in the Boolean model $C_{\text {micro }}(h)$ and of the mean stress field $C_{\sigma_{m}}(h)$, estimated from FFT computations. In the case of the characteristic phase, the theoretical covariance of the Boolean model $Q(h)=P\left\{x \in A^{c}, x+h \in A^{c}\right\}$ is given by :

$$
Q(h)=q^{2-r(h)},
$$

where $r(h)=\frac{K(h)}{K(0)}, K(h)$ being the geometrical covariogram of the primary grain $A^{\prime}$ of the model (here a sphere with radius $a$ ). In the present case we have:

$K(h)=\bar{V}\left(A^{\prime} \cap A_{-h}^{\prime}\right)=\frac{\pi}{6}(2 a)^{3}\left[1-\frac{3}{2} \frac{h}{2 a}+\frac{1}{2}\left(\frac{h}{2 a}\right)^{3}\right]$ for $h<2 a$, else $K(h)=0$

Starting from Eqs. (13) and (16), the integral range of the volume fraction of the Boolean model is easily computed as a function of $p$ or $q$. Alternatively, it can be estimated from the simulations of the microstructure, which gives a validation of the representativity of the simulations.

When a hydrostatic loading is applied, the function $C_{X}(h)$ does not depend on the direction of the vector $h$ and is approximated by computing the mean of the three values $C_{X}\left(|h| e_{1}\right), C_{X}\left(|h| e_{2}\right)$ and $C_{X}\left(|h| e_{3}\right)$ in the three privileged directions of the FFT grid.

In this section we also make use of stress field maps as shown in Figs. (12) and (13). They represent random $2 \mathrm{D}$ sections of the mean component of the stress field $\sigma_{m}$, for the porous and rigid Boolean models, respectively, at various increasing volume fractions $f$ of the spheres. Colors have been adjusted to the maximum and minimum of the field in each section to highlight the field patterns. A detailed study of these fields is out of the scope of the present paper.

Plotted as a function of the distance $h$, the covariance function exhibits a characteristic length of the microstructure. For a continuous field, it makes conspicious the typical length related to fluctations of the apparent average of a field over subdomains. Applied to the mean stress field, this is the minimal length of the representative volume element. Normalizing the covariance as $\widetilde{C}(h)=\left[C(h)-\sigma_{0}^{2}\right] /\left[C(0)-\sigma_{0}^{2}\right]$, covariance functions $\widetilde{C}(h)$ of the mean stress are plotted as a function of the length $h$, for various concentrations $f$ of the rigid phase in Fig. 8. From this figure it appears that the correlation length (or range) of the mean component of the stress field (i.e. the length for which the central correlation function reaches its asymptotic value 0) compares well to the diameter of the spheres (18 voxels in the present case) for the lower 
pore volume fraction $(f=0.25)$. For higher porosities, the range of the stress field is larger than the range of the microstructure.

Normalized covariance functions $\widetilde{C}(h)$ of the mean stress in a Boolean model with spherical rigid inclusions are given in Fig. (7). Contrary to the porous case, it is observed that the correlation length of the mean component of the stress field is significantly higher to the diameter of the spheres except for volume fractions $f$ lower than the percolation threshold.

The integral ranges obtained from the numerical simulations (using the procedure explained in subsection 6.2.1) are given as a function of the volume fraction of inclusions $f$ in Fig. (9. They are normalised for a sphere radius $a$ equal to 1 . Error bars are obtained from the quality of the fit obtained by means of the theroretical change of the variance $D_{Z}^{2}(V)$ with the volume $V$ (Eq. 12).

It appears that the integral range (and therefore the RVE) of the microstructure is smaller than that of the mean stress component in the composites, except in regimes where the softer phase has percolated or is close to the percolation threshold (when $f>0.8$ and $f<0.35$, for the porous and rigidlyreinforced media, respectively).

For porosities lower than the percolation threshold, the integral range of the mean stress $\sigma_{m}$ is close, but slightly larger than that of the microstructure. As expected, high values of the mean stress component are concentrated around voids (see e.g. maps (b) and (c) in Fig. 12), inducing anti-correlations and lowering the integral ranges.

Such phenomenon is not observed in the rigidly-reinforced media, where the stress field in the rigid inclusions is high. In this material, the integral range of the mean stress is, consequently, much larger than that of the microstructure beyond the percolation threshold. Such large integral ranges have already been observed $(13 ; 14)$ for another model of microstructure, in cases of finite contrast between the phases.

\subsection{Field histogram}

This section is concerned with the field histograms of the hydrostatic stress component. The field histogram (i.e. probability distribution) for the mean stress in the matrix (phase 1) is defined as:

$$
P_{\sigma_{m}}(t)=\frac{1}{1-f} \int_{(1)} \mathrm{d}^{2} \mathbf{x} \delta\left(\sigma_{m}(\mathbf{x})-t\right)
$$


Field histograms are computed numerically by means of the FFT solutions, and are plotted in Figs. (10) and (11), for the porous and rigid media respectively. Each figure shows a set of curves corresponding to particular values of the concentration $f$ of the spheres. As in the previous section, we appeal to maps of the hydrostatic stress component as given in Figs. (12) and (13), for porous and rigid Boolean models, respectively, to discuss the effects of the morphology of the microstructure on the local distribution of strains and of stresses in the material.

In general, the field distributions $P_{\sigma_{m}}$ of the mean stress component are not Gaussian, and non-symmetric. In the porous material, the field distribution $P_{\sigma_{m}}$ (Fig. 10) takes high values around the average of the mean stress in the matrix $\sigma_{m}=\left\langle\sigma_{m}\right\rangle_{(1)}$ for $f<0.3$. For higher porosities, large values of the distribution are found at $\sigma_{m}=0$. At a particular volume fraction $f \approx 0.7$ (see e.g. map (d) in Fig. 12), one of the local maxima of $P_{\sigma_{m}}$ disappears, at which point the distribution field is highly non-symmetric. This change of the shape of the distribution corresponds to the appearance of "dead zones" of the matrix where the strain and stress fields are null.

In the rigid material, however, the distribution $P_{\sigma_{m}}$ of the mean stress in the matrix, as shown in Fig. (11), always has a unique maximum. A change of the shape of the distribution is observed near $f=0.3$, close to the percolation threshold. At this volume fraction, it is conspicuous that the distribution $P_{\sigma_{m}}$ is most non-symmetric, whereas for $f>0.3$, when the "matrix" is made of isolated clusters and is much softer than the spheres, the distribution $P_{\sigma_{m}}$ is close to a Gaussian distribution.

\section{Conclusion}

The coupling between 3D simulations of random sets and the numerical resolution of the equations of elasticity by means of FFT iterations is a powerful tool to estimate the effective properties of random media, even in the case of a high contrast between its components.

Using this methodology, we could, for the first time, estimate the variation of the bulk modulus of Boolean models of spheres in the case of porous or rigid inclusions. In addition to the effective properties, fluctuations of the field averages over finite domains and histograms of the mean component of the stress field were investigated. The same approach may be applied to multiscale heterogeneous materials (12), and to multi-component media, allowing a comparison with the prediction of standard models, such as the self-consistent estimates or bounds of effective properties. 
The representative volume element (RVE) depends on the properties under consideration. For porous media, for most volume fractions, the size of the RVE for the mean stress component is close to that of the RVE of the microstructure. It is either smaller, or much larger, in the case of rigidly-reinforced media. For porous materials, bimodal distributions of the mean stress were observed. Investigations regarding the shear modulus of Boolean models are currently undertaken using similar numerical techniques.

The authors are indebted to Institut Carnot M.I.N.E.S. for a financial support during this study. 


\section{References}

[1] M.J. Beran, J. Molyneux, Use of classical variational principles to determine bounds for the effective bulk modulus in heterogeneous media, Q. Appl. Math. 24 (1966), 107-118.

[2] D. J. Bergman, The dielectric constant of a composite material-A problem in classical physics, Phys. rep. 43, 9 (1978), 377-407.

[3] I. Cohen, D. J. Bergman, Clausius-Mossotti-type approximation for elastic moduli of a cubic array of spheres, Phys. Rev. B 68 (2003), 024104.

[4] I. Cohen, Simple algebraic approximations for the effective elastic moduli of cubic arrays of spheres, J. of Mech. and Phys. of Sol. 52, 9 (2004).

[5] A. Delarue, Prévision du comportement électromagnétique de matériaux composites à partir de leur mode d'élaboration et de leur morphologie, Thesis, Paris School of Mines (2001).

[6] D. Deptuck, J. P. Harrison, P. Zawadzki, Measurement of elasticity and conductivity of a three-dimensional percolation system, Phys. Rev. Lett. 54 (1985), 913.

[7] W. T. Elam, A. R. Kerstein, J. J. Rehr, Critical properties of the void percolation problem for spheres, Phys. Rev. Lett. Vol. 52, N. 17 (1984), 1516-1519.

[8] D. J. Eyre, G. W. Milton, A fast numerical scheme for computing the response of composites using grid refinement, Eur. Phys. J. AP. 6 (1999), 1.

[9] Z. Hashin, S. Shtrikman, A variational approach to the theory of the elastic behavior of multiphase materials, J. of the Mech. and Phys. of Sol. 11 (1963), 127-140.

[10] D. Jeulin, M. Ostoja-Starzewski (eds) Mechanics of Random and Multiscale Microstructures, CISM Lecture Notes N 430 (2001), Springer Verlag.

[11] D. Jeulin, Random Structures in Physics, in: "Space, Structure and Randomness", Contributions in Honor of Georges Matheron in the Fields of Geostatistics, Random Sets, and Mathematical Morphology, Series: Lecture Notes in Statistics, Vol. 183, M. Bilodeau, F. Meyer, M. Schmitt (eds) (2005), XIV, Springer-Verlag, 183-222. strongly anisotropic,

[12] D. Jeulin, M. Moreaud, Percolation d'agrégats multi-échelles de sphères et de fibres: Application aux nanocomposites, in Matériaux 2006, Dijon.

[13] D. Jeulin, M. Moreaud Statistical representative volume element for predicting the dielectric permittivity of random media, Proc. CMDS 11, D. Jeulin \& S. Forest (eds) Les Presses de l'École des Mines de Paris (2008), 429-436.

[14] T. Kanit, S. Forest, I. Galliet, V. Mounoury, D. Jeulin, Determination of the size of the representative volume element for random composites: statistical and numerical approach, Int. J. of Sol. and Str. 40 (2003).

[15] T. Kanit, F. N'Guyen, S. Forest, D. Jeulin, M. Reed, S. Singleton, Apparent and effective physical properties of heterogeneous materials: Rep- 
resentativity of samples of two materials from food industry, Comput. Methods Appl. Mech. Engrg. 195 (2006), 3960.

[16] E. Kroner, Statistical Continuum Mechanics, Springer-Verlag, Wien (1972).

[17] G. Matheron, The theory of regionalized variables and its applications, Paris School of Mines publications (1971).

[18] G. Matheron, Random sets and integral geometry, J. Wiley, New York (1975).

[19] G. Matheron, Estimating and Choosing, Springer Verlag, Berlin (1989).

[20] J.-C. Michel, H. Moulinec, P. Suquet, A computational scheme for linear and non-linear composites with arbitrary phase contrast, Int. J. Numer. Meth. Engng. (2001).

[21] G. Milton, Bounds on the elastic and transport properties of two component composites, J. Mech. Phys. Solids, 30 (1982), 177-191.

[22] H. Moulinec, P. Suquet, C. R. Acad. Sci. Paris II 318 (1994), 1417.

[23] for most volume fractions H. Moulinec, P. Suquet, A numerical method for computing the overall response of nonlinear composites with complex microstructure, Comp. Methods in Appl. Mech. and Engrg. 157 (1998), $1-2$.

[24] H. Moulinec, P. Suquet, Comparison of FFT-based methods for computing the response of composites with highly contrasted mechanical properties, Phys. B, 338 (2003).

[25] M. D. Rintoul, S. Torquato, Precise determination of the critical threshold and exponents in a three-dimensional continuum percolation model, J. Phys. A.: Math; gen. 30 (1997).

[26] S. Torquato, Random heterogeneous media: microstructure and improved bounds on effective properties, Appl. Mech. Rev., 44 (1991), 37-76.

[27] F. Willot, Y.-P. Pellegrini, P. Ponte Castañeda, Localization of elastic deformation in strongly anisotropic, porous, linear materials with periodic microstructures: Exact solutions and dilute expansions, J. of the Mech. and Phys. of Sol. 56 (2008), 4. 


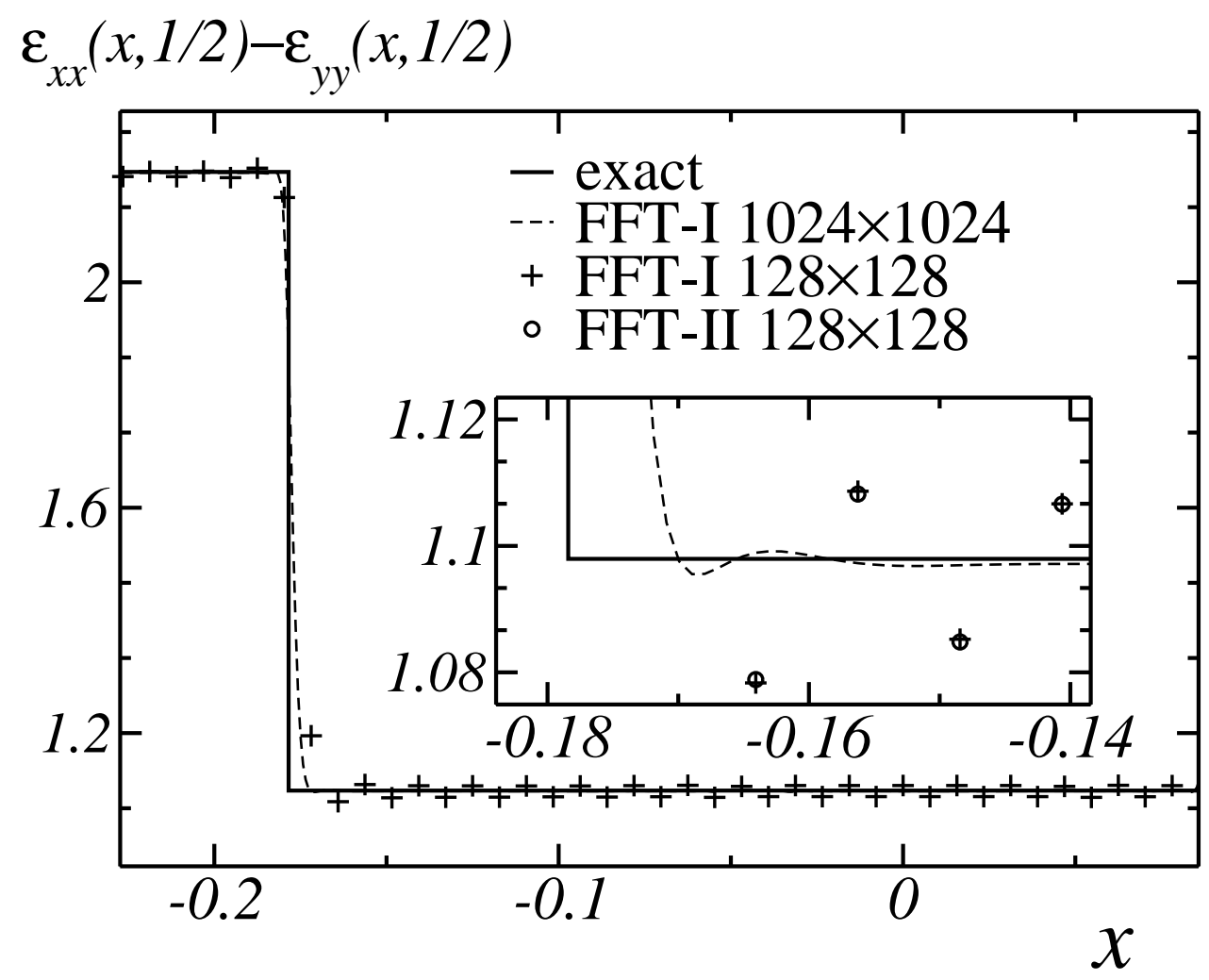

Fig. 1. Difference os the strain components $\varepsilon_{x x}-\varepsilon_{y y}$ along a half-line $y=1 / 2$ located on the border of the unit cell, for a periodic array of cylinders: comparison between the exact solution in the continuum (solid line) and two FFT methods, "FFT-I" and "FFT-II" with slightly different treatment of the highest frequencies. Two grid sizes are shown for the first method. The material is strongly anisotropic (see Willot, Pellegrini, Ponte (27)) and "pure shear" loading applied (i.e. only the macroscopic components $\left\langle\varepsilon_{x x}\right\rangle=-\left\langle\varepsilon_{y y}\right\rangle$ are non zero). 


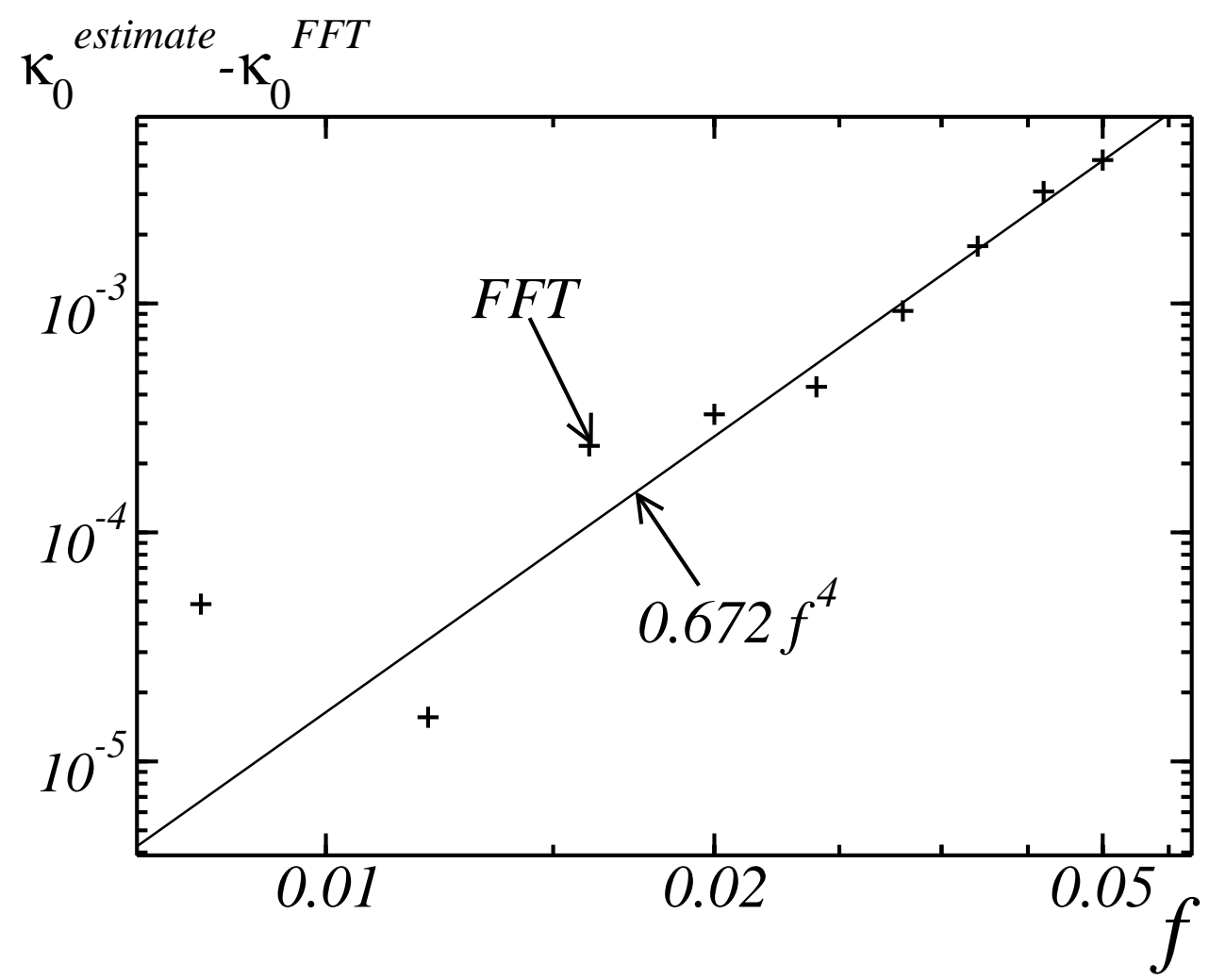

Fig. 2. Difference $(+)$ between the Bergman estimate and FFT results for the effective bulk modulus $\kappa_{0}$ in a periodic array of spheres as a function of the concentration of spheres, in log-log scale. The spheres have elastic moduli that are two times higher than in the matrix. The error $(+)$ is of order $\sim f^{4}$ when $f$ is small (solid line). 


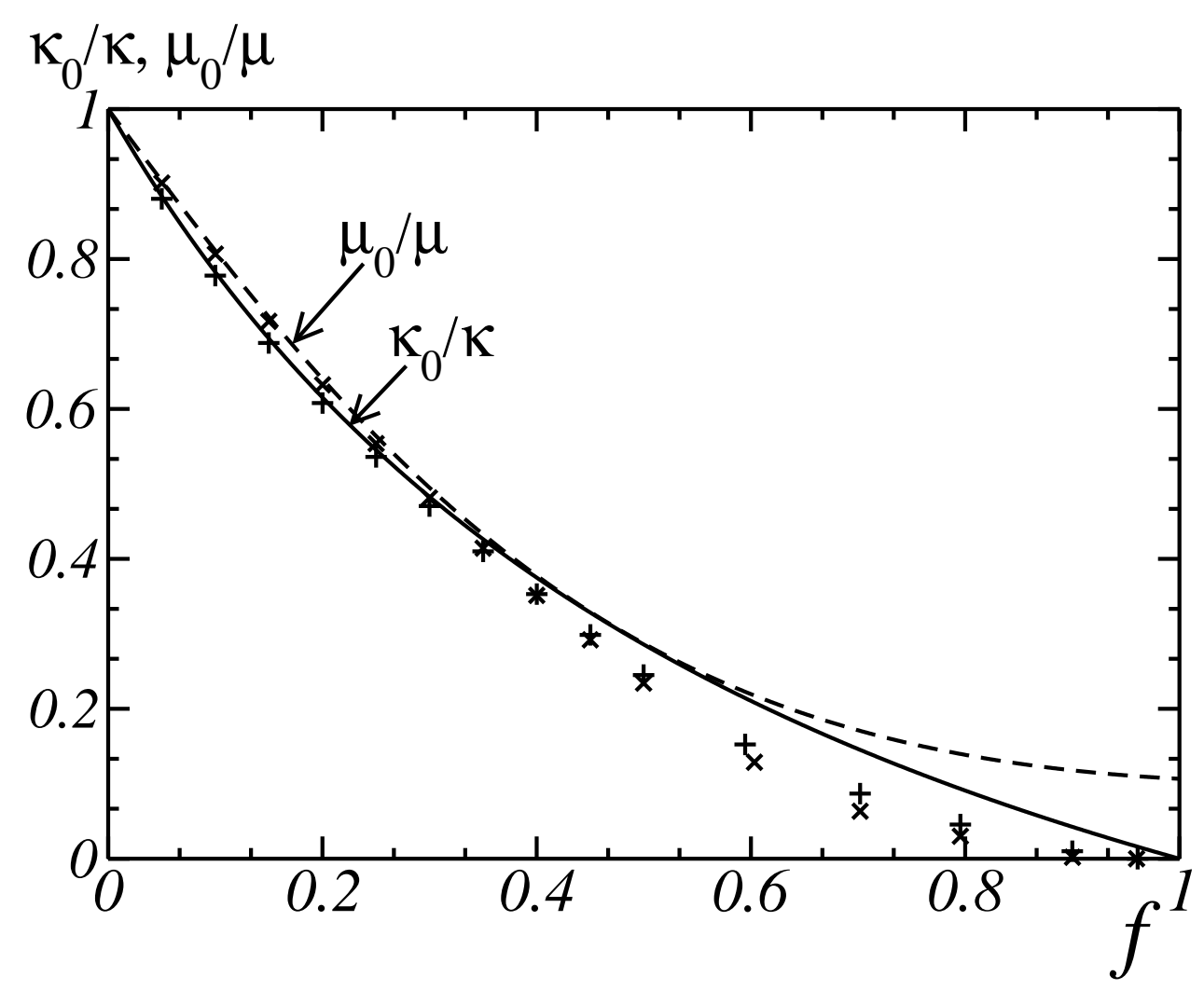

Fig. 3. Comparison between Bergman estimates for the effective bulk modulus $\kappa_{0}$ (solide line), the effective shear modulus $\mu_{0}$ (dotted line) and FFT computations (symbols + and $\times$, respectively) of a periodic array of spherical voids. 


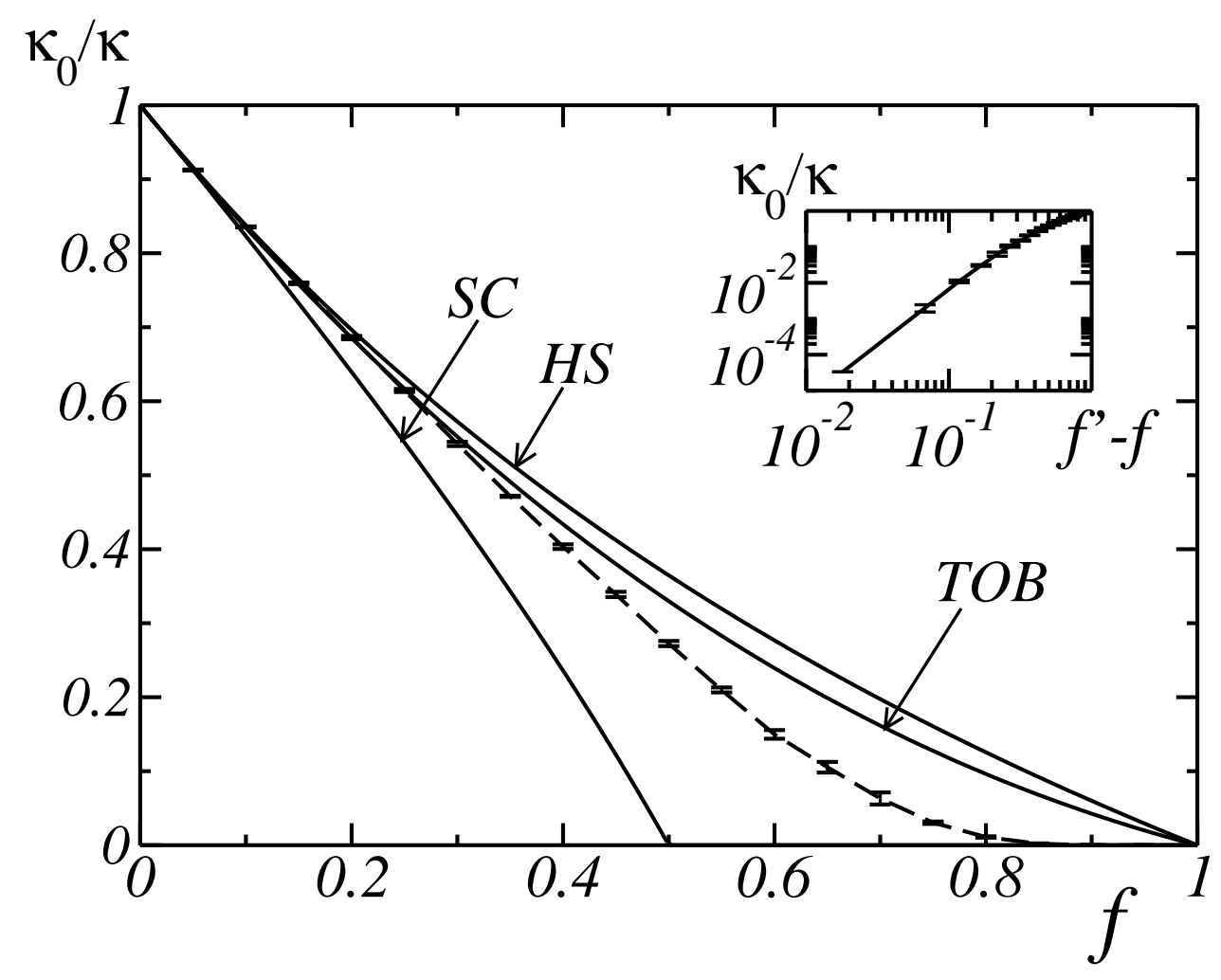

Fig. 4. normalized effective bulk modulus $\kappa_{0} / \kappa$ as a function of the porosity $f$ : FFT computations (10 samples on $256^{3}$ grids), self-consistent estimate (SC), upper Hashin-Shtrikman (HS) and third-order bound (TOB), solid lines. 


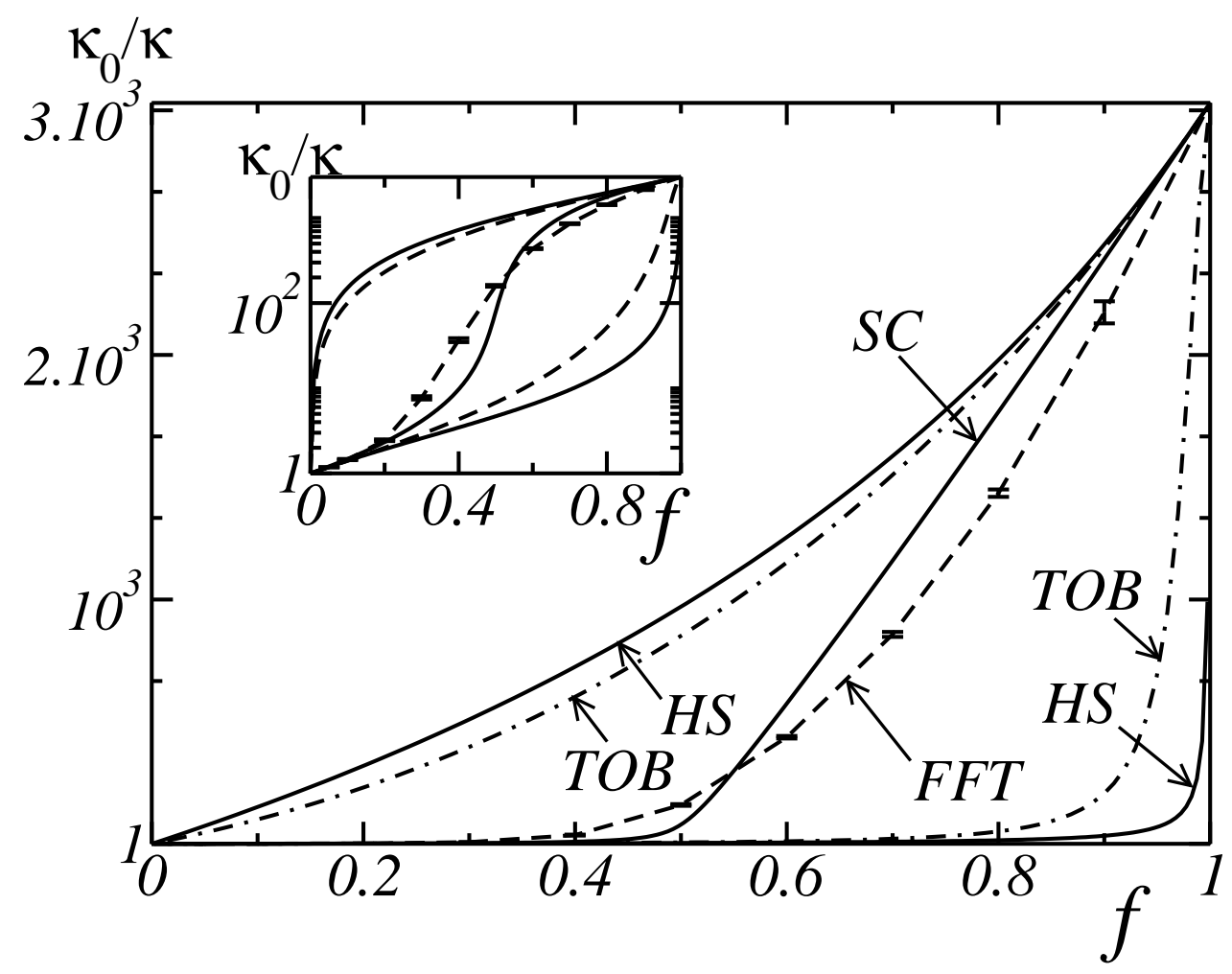

Fig. 5. normalized effective bulk modulus $\kappa_{0} / \kappa$ as a function of the rigid particles volume fraction: FFT computations (10 samples on $256^{3}$ grids), self-consistent estimate (SC), upper and lower Hashin-Shtrikman (HS) and third-order bounds (TOB), solid lines. 


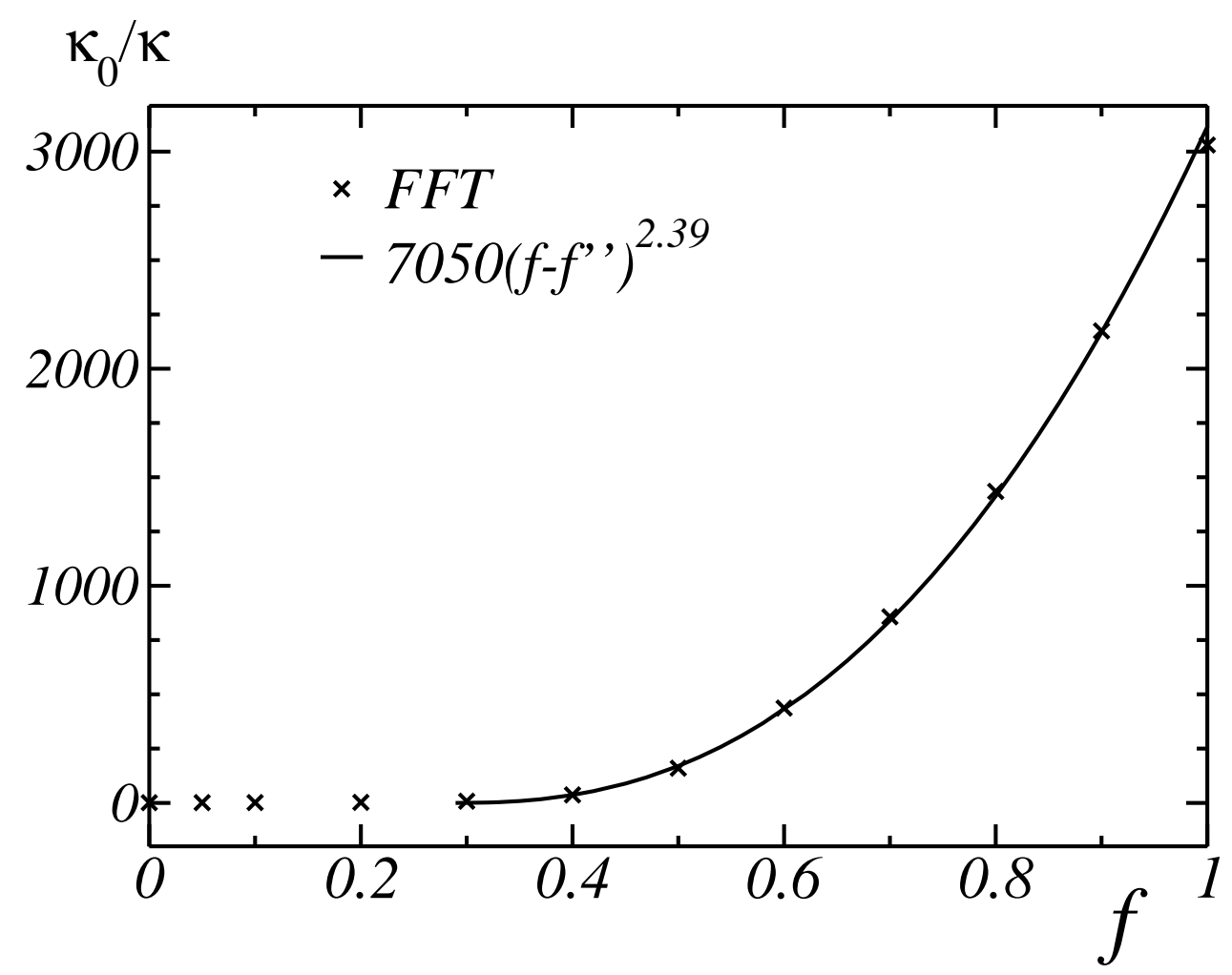

Fig. 6. Normalized effective modulus $\kappa_{0} / \kappa$ computed by FFT $(+)$ for a rigidly-reinforced Boolean model of spheres as a function of the concentration of particules $f$, and a powerlaw fit of these values in the regime $f>f^{\prime \prime}$ (solid lines), where spheres have percolated. 


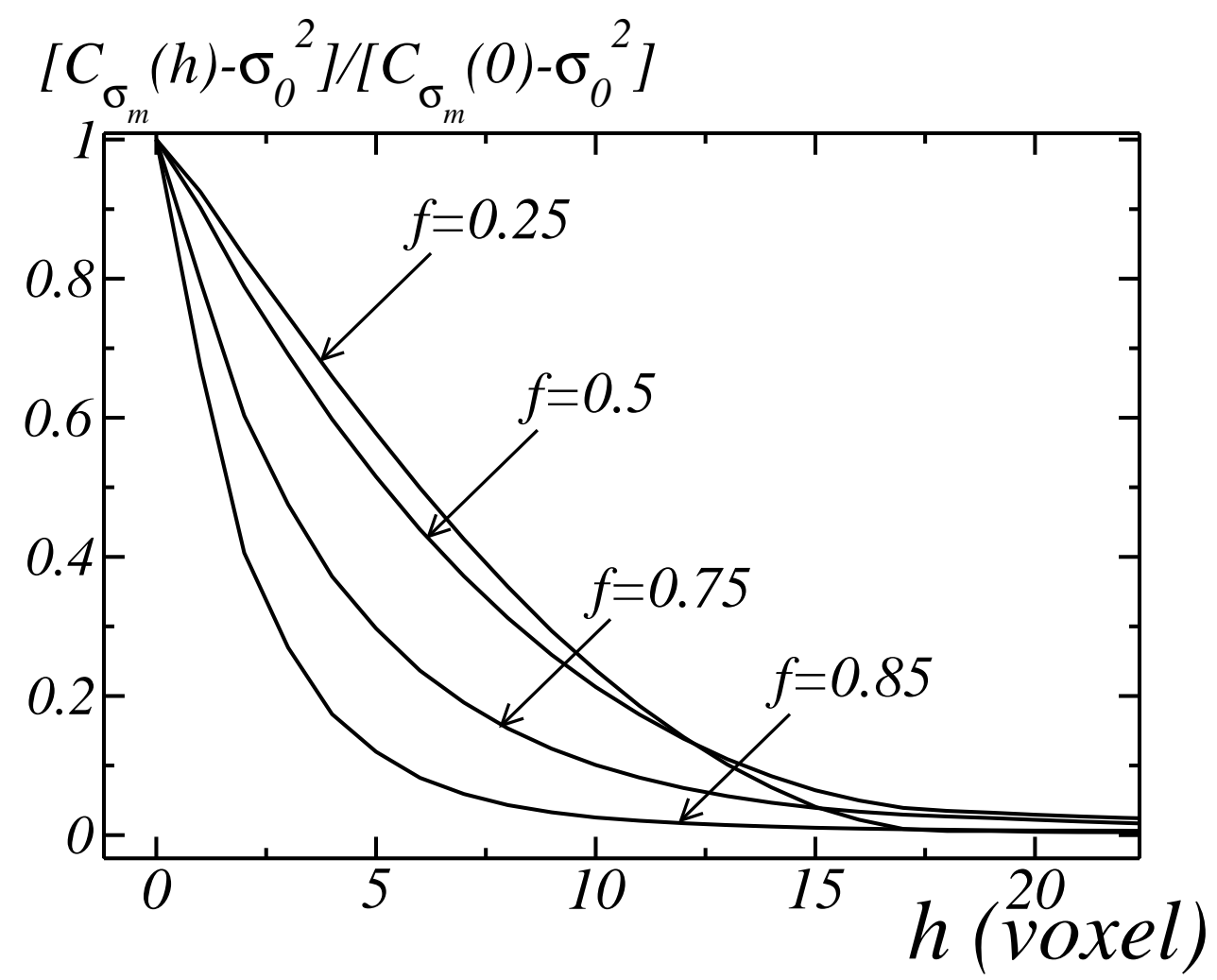

Fig. 7. Normalized covariance $C_{\sigma_{m}}(h)$ of the mean component of the stress field $\sigma_{m}$ as a function of the distance $h$ (number of voxels), for a Boolean medium at various different porosities $f=0.25,0.5,0.75$ and 0.85 . Along the $x$-axis, the length $h$ is expressed in voxel units (the diameter of a sphere is 18 voxels). A hydrostatic load is applied as a macroscopic deformation $\left\langle\varepsilon_{m}\right\rangle=1$, on a $256^{3}$ FFT grid. 


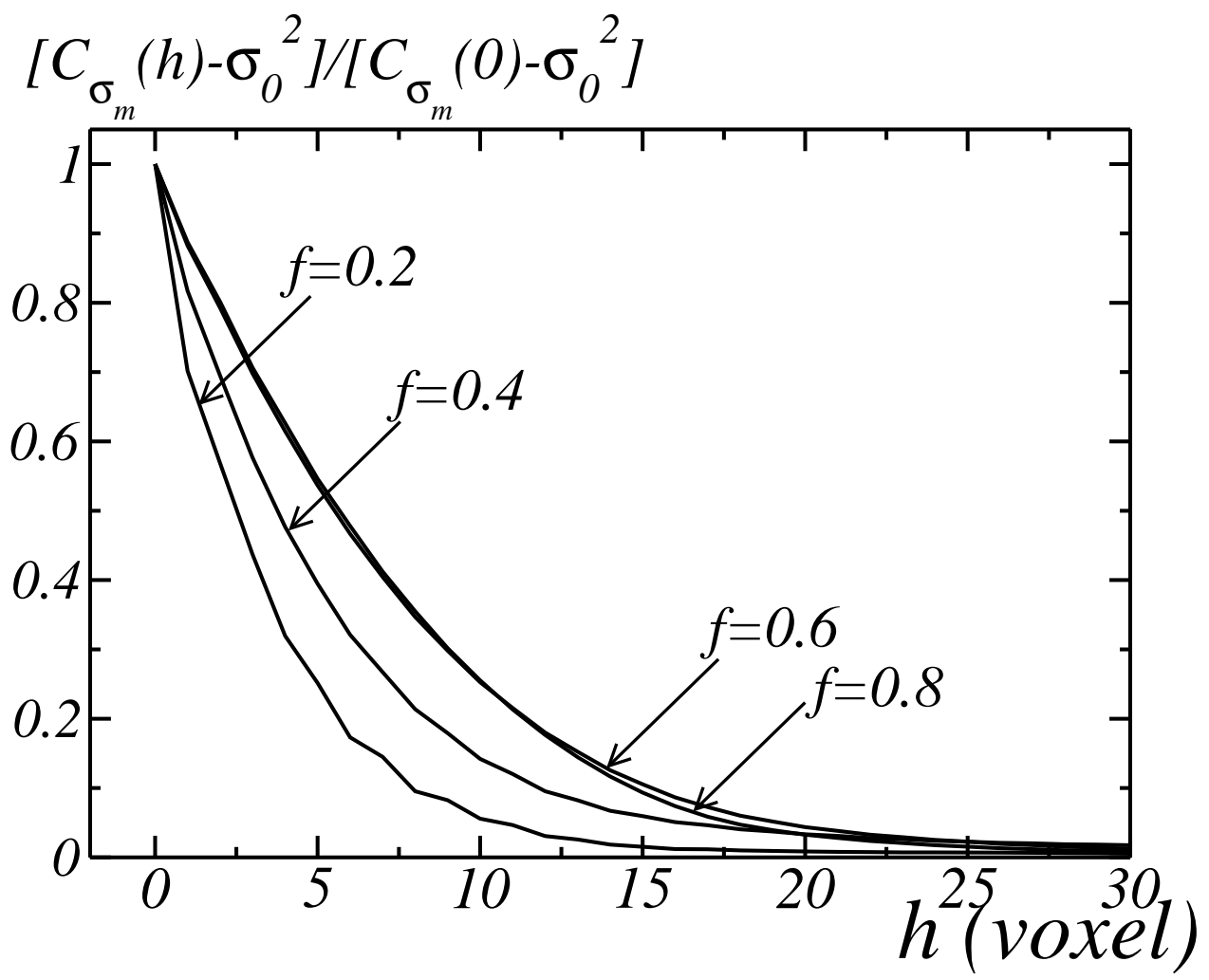

Fig. 8. Normalized covariance $C_{\sigma_{m}}(h)$ of the mean component of the stress field $\sigma_{m}$ as a function of the distance $h$ (number of voxels), for a Boolean medium at various different concentration of rigid inclusions $f=0.2,0.4,0.6$ and 0.8. Along the $x$-axis, the length $h$ is expressed in voxel units (the diameter of a sphere is 18 voxels). A hydrostatic load is applied as a macroscopic deformation $\left\langle\varepsilon_{m}\right\rangle=1$, on a $256^{3}$ FFT grid. 


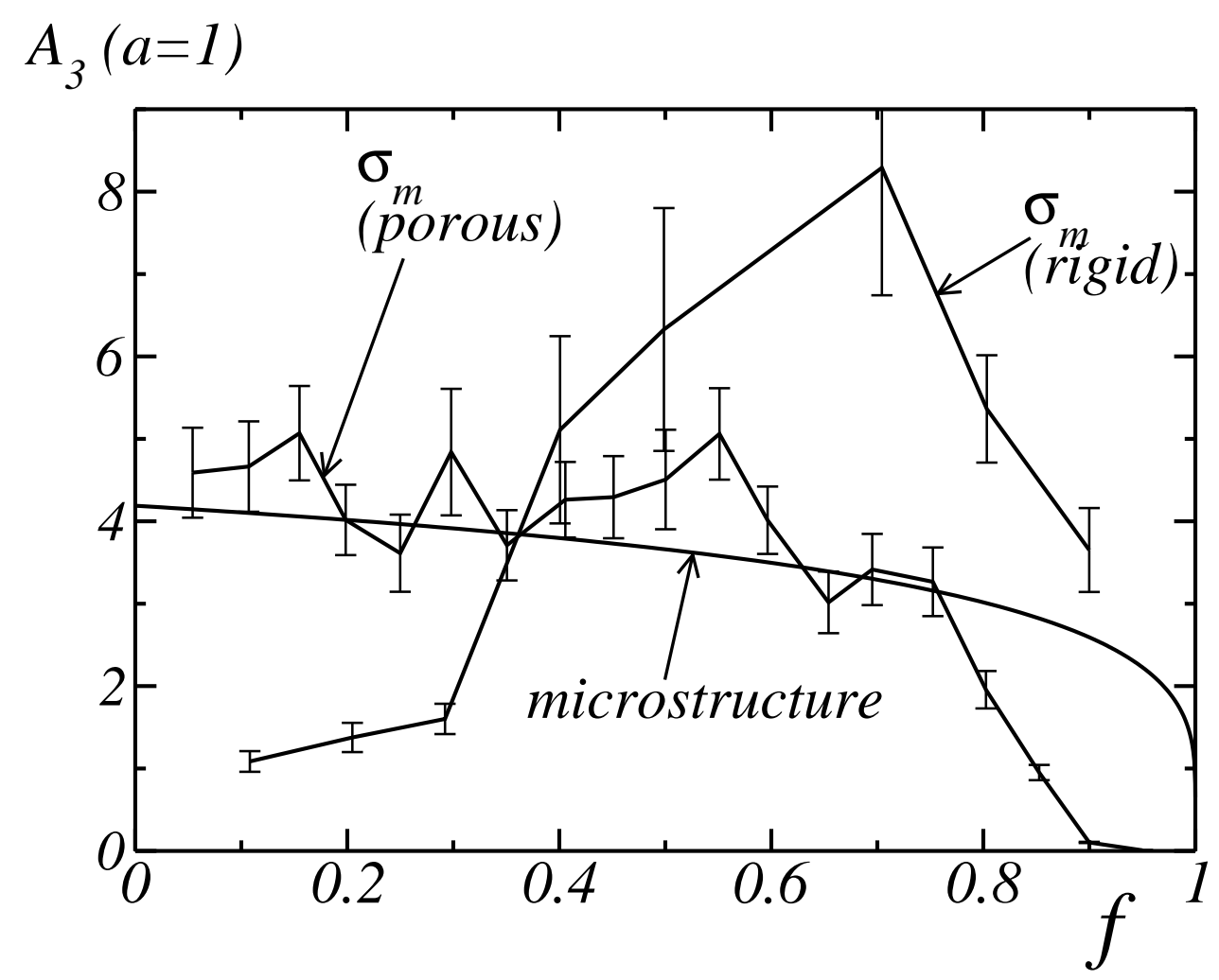

Fig. 9. Integral range of the mean component of the stress field $\sigma_{m}$, in the random porous as well as rigidly-reinforced media, and of the Boolean microstructure as a function of the porosity $f$. Values on the $y$-axis correspond to a sphere radius $a=1$ 


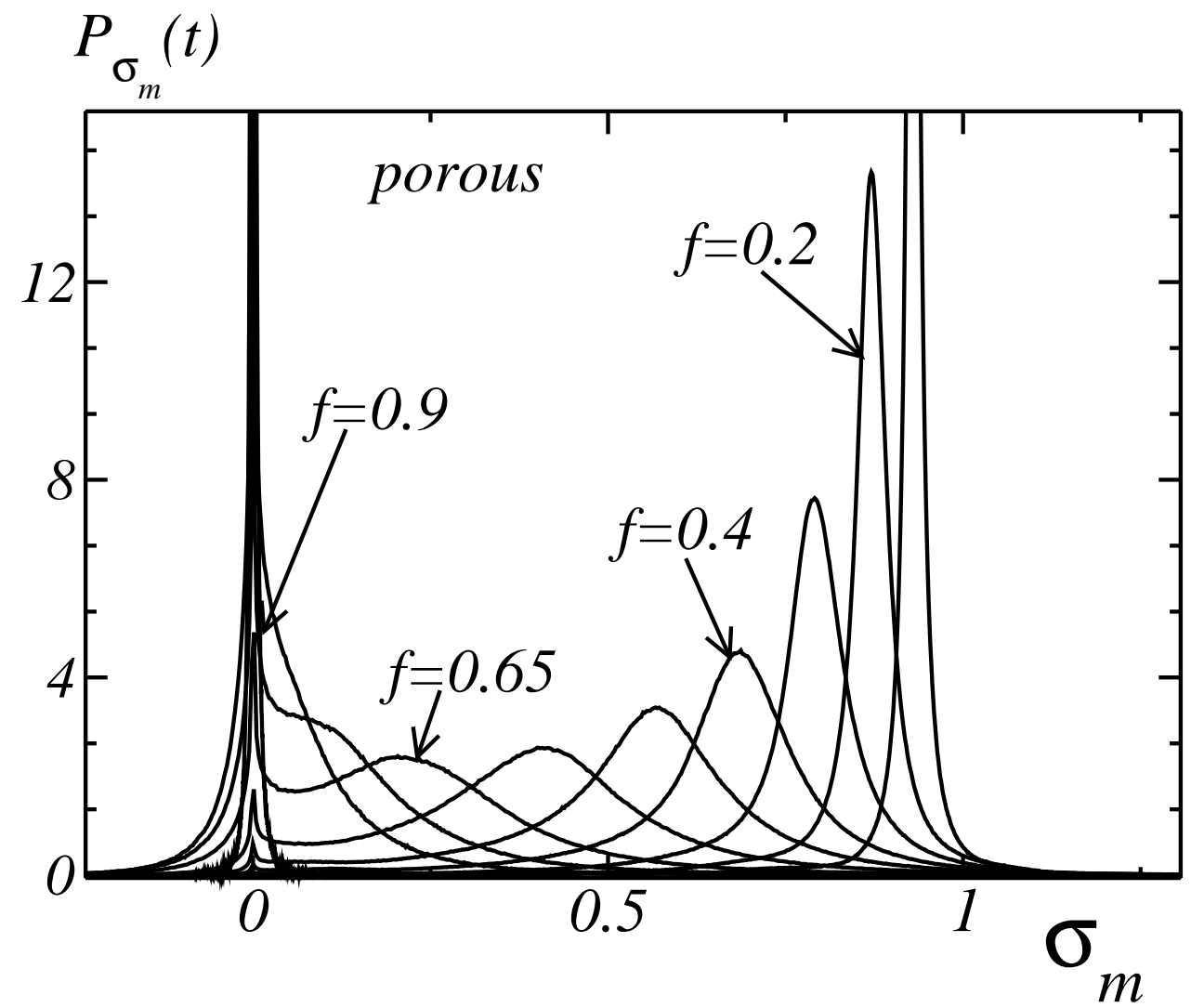

Fig. 10. Histograms $P_{\sigma_{m}}(t)$ of the mean component of the stress field $\sigma_{m}$ in the matrix, in the porous random medium at increasing porosities $f=0.1,0.2,0.3,0.4$, $0.5,0.6,0.65,0.7,0.8,0.9$ (right to left, as indicated on the figure). The values of the stress on the $x$-axis correspond to a hydrostatic macroscopic deformation $\left\langle\varepsilon_{m}\right\rangle=1$, on a $256^{3}$ FFT grid. 


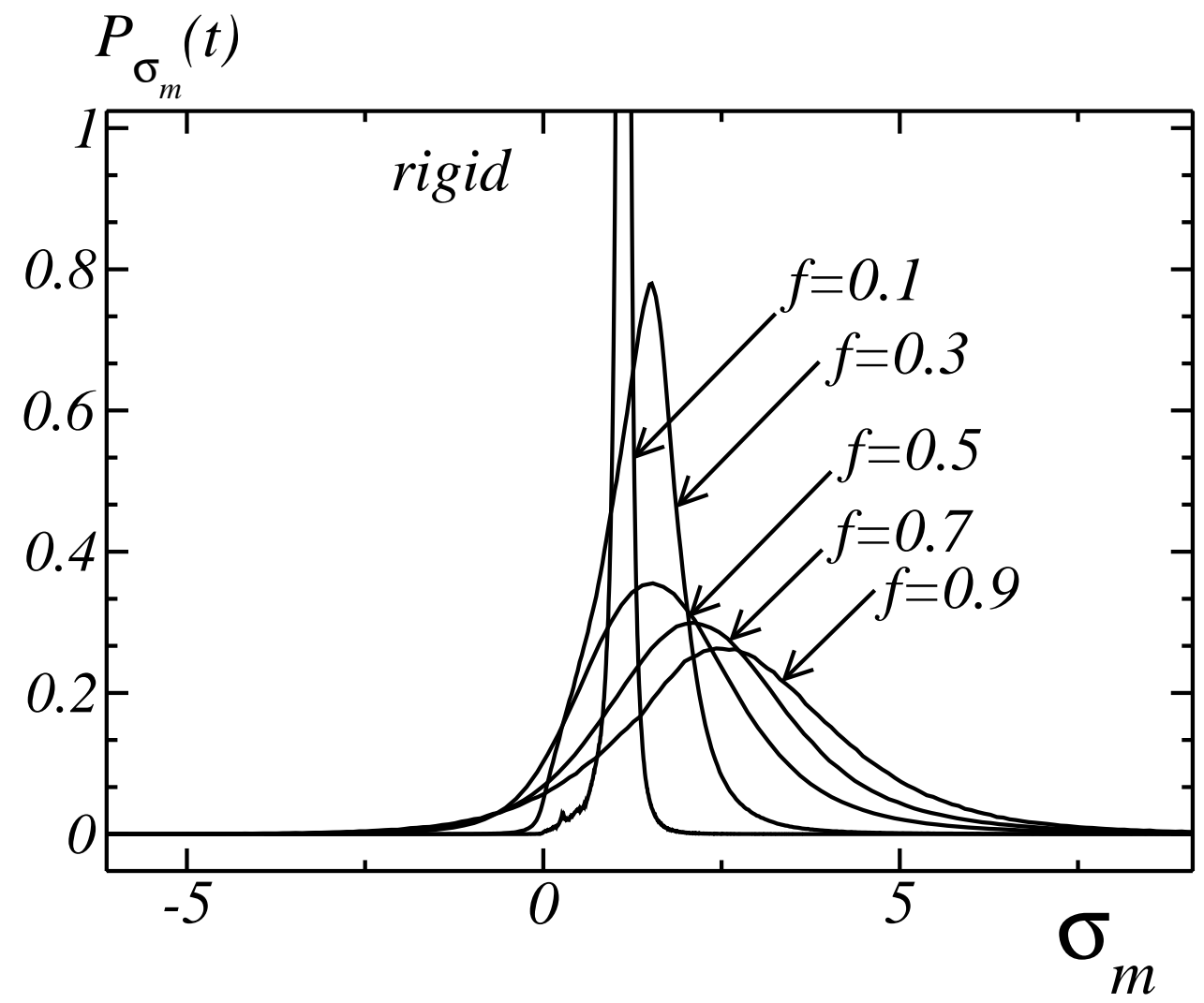

Fig. 11. Histograms $P_{\sigma_{m}}(t)$ of the mean component of the stress field $\sigma_{m}$ in the matrix, in the rigidly-reinforced random medium at increasing volume fractions of the rigid particles $f=0.1,0.2,0.3,0.4,0.5,0.6,0.65,0.7,0.8,0.9$ (right to left, as indicated on the figure). The values of the stress on the $x$-axis correspond to a hydrostatic macroscopic deformation $\left\langle\varepsilon_{m}\right\rangle=1$, on a $256^{3}$ FFT grid. 


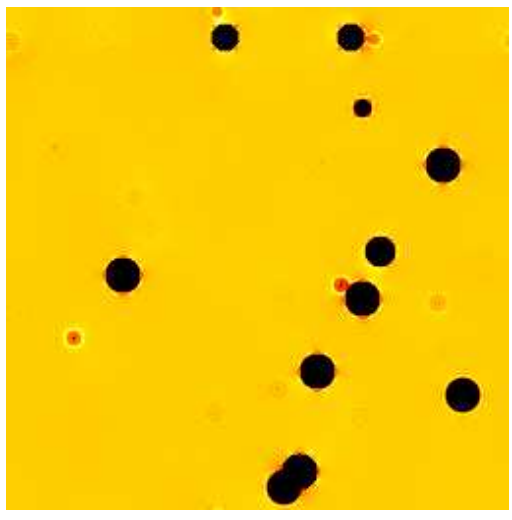

(a)

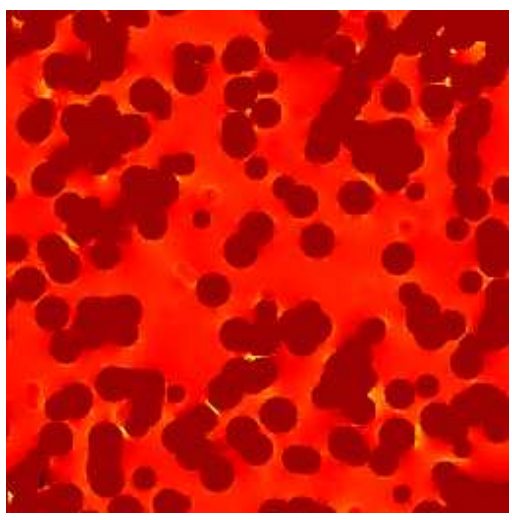

(c)

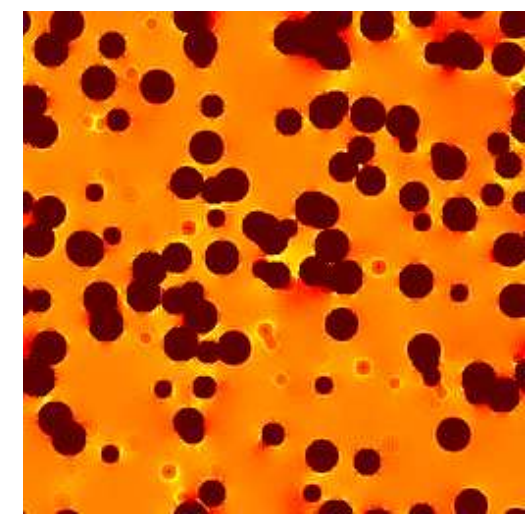

(b)

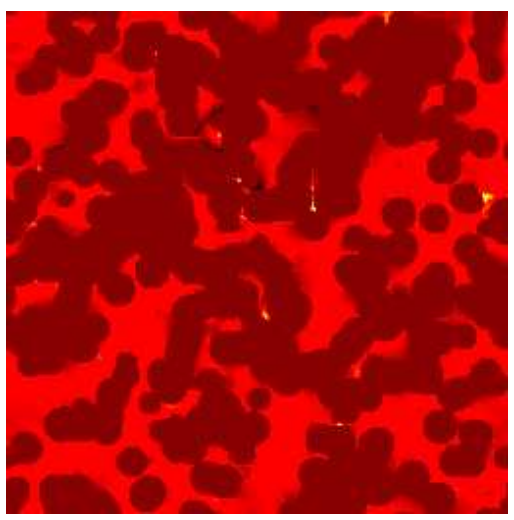

(d)

Fig. 12. 2D sections of the stress component $\sigma_{m}$ in the random porous medium, at various porosities $f=0.05$ (a), 0.25 (b), 0.45 (c), 0.6 (d). A hydrostatic deformation $\left\langle\varepsilon_{m}\right\rangle$ is applied. The color scale goes from black (smallest values) to white (highest values) with red and yellow in-between (color online). 


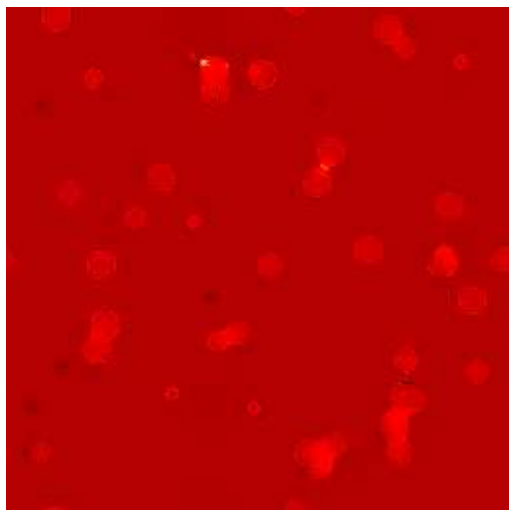

(a)

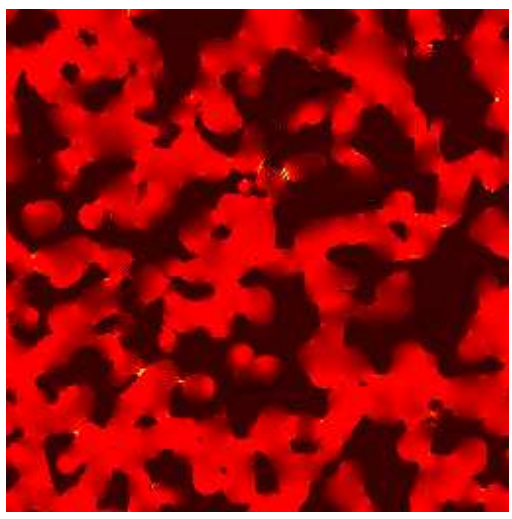

(c)

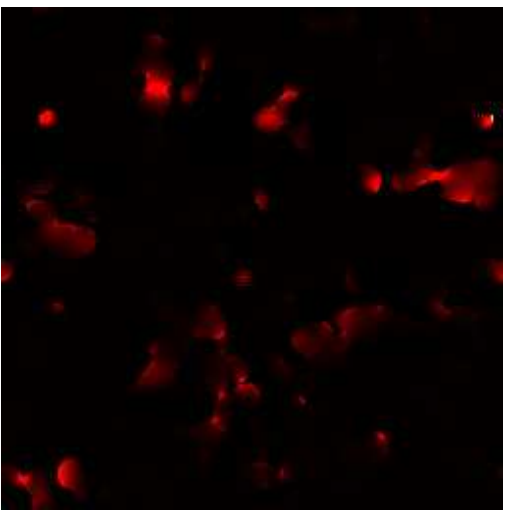

(b)

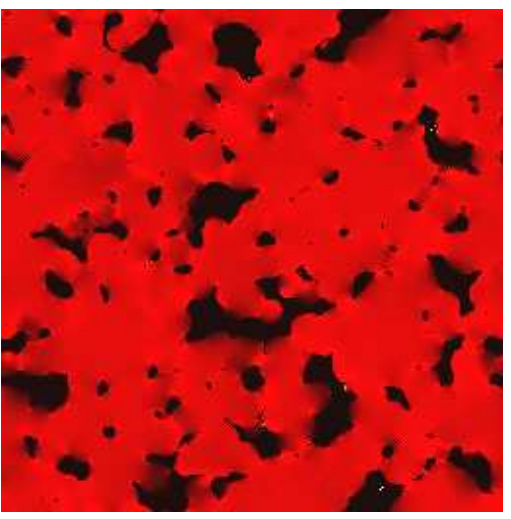

(d)

Fig. 13. 2D sections of the stress component $\sigma_{m}$ in the random rigidly-reinforced medium, at various rigid inclusions concentrations $f=0.1$ (a), 0.4 (b), 0.7 (c), 0.9 (d). A hydrostatic deformation $\left\langle\varepsilon_{m}\right\rangle$ is applied. The color scale goes from black (smallest values) to white (highest values) with red and yellow in-between (color online). 